\title{
Inadequacy of death certification: Proposal for change
}

\author{
T G Ashworth
}

\section{Introduction}

The inaccuracies of death certification and the relevance of post mortem examination in redressing error have been the subject of an illuminating book. ${ }^{1}$ Contrary to popular belief these inaccuracies have not been eradicated with the advent of sophisticated modern technology. ${ }^{23}$ Minerva, in the British Medical Journal, reported that the Journal of the American Medical Association no longer included causes of death in obituaries because the sources of information were not reliable enough. ${ }^{4}$

Major public health decisions reflected in government policies are based on these vital statistics. We are constantly bombarded, with missionary zeal, by advocates for one form of lifestyle or another, based on the same information. Many authors in the United Kingdom have pointed out that the necropsy provides the only valid means of correcting these inaccuracies. ${ }^{5-7}$ In the United States journals such as Human Pathology and Archives of Pathology and Laboratory Medicine have devoted most of their issues to the subject. ${ }^{89}$ The importance of necropsies in clinical audit should not be disputed. ${ }^{10}$ It has, however, taken three issues of The National Confidential Enquiry into Perioperative Deaths (NCEPOD) for the pathological findings to be given their proper place. ${ }^{11}$

The value of the necropsy is only as good as the answers it provides, and many may be hidden in the depths of a well constructed essay. Collectors of statistics do not have this insight, merely the listed causes of death with possible additional information in the box for those conditions, "contributing to the death but not related to the disease or condition causing it."

The major potential source of accurate information in England and Wales is provided by Coroners' necropsies, 134879 having been performed in 1987, an impressive number by any standards: 13764 were also performed for medical interest. With 566994 deaths in England and Wales in 1987, the overall necropsy rate was $26 \%$. We can ill afford not to maximise this combined effort. The public may be satisfied by most aspects of the Coroners' system and the Coroners themselves are well served by the results in apportioning deaths due to natural and unnatural causes, but it is my opinion that the morbidity and mortality statistics that arise from this large body of information are seriously flawed, mainly because of the omission of important material.
While there are other aspects of the Coroners' system that need to be examined, such as the unequal distribution of necropsies among pathologists, the necessity of London based forensic practitioners to perform vast numbers to fund their departments, and the widely divergent attitudes to the necropsy and its report in general among pathologists and Coroners alike, this paper addresses one area-that of death certification.

Fundamental to the issue is the anachronism that only those factors that are thought to be directly related to death are included in certification. In practice, deciding what should or should not be included may prove extremely difficult and tends to become a matter of opinion and not fact, which it purports to be. Indeed, I have long held the view that what needs to be recorded is not the "diseases or conditions directly leading to death," but with what diseases or conditions did death occur. Those conditions that are not directly related to death, but might well be of vital importance to public health, may not be included in the present death certification.

The following are but a few examples:

(1) Incidental carcinomas and other tumours, peptic ulcers, gallstones, colonic adenomata, etc.

(2) The severity of atheroma, cerebral, coronary and aortic.

(3) A normally functioning artificial heart valve, inserted 10 years previously.

(4) A transplanted kidney with no apparent contribution to the cause of death.

(5) Diabetes mellitus.

(6) Malignancy treated years before, of which there is no evidence at death.

Other examples are mentioned below. These conditions may well be in the framework of the post mortem report, but are filed in patients' notes and Coroners' offices up and down the land and never seen again. It is not even universal practice to send copies, where appropriate, of Coroners' necropsy reports to clinicians or cancer registries.

One of the essentials of medical audit is to use resources adequately and effectively. It is suggested that the information derived from the present death certificate fails on both counts. With a little extra time devoted to death certification, and particularly to the necropsy, valuable material for present and future generations could be preserved.

What follows could well be applied in part to death certification where a necropsy has not been performed, but where disease processes,

\author{
Correspondence to: \\ T G Ashworth \\ Accepted for publication \\ 16 August 1990 \\ Pathology, Walsgrave \\ Hospital, Coventry \\ T G Ashworth
}


incidental to the cause of death, are known and documented.

\section{The proposal}

An addendum to be accommodated on the back of the present death certificate, the Coroners' form 100 "Notification to the Registrar of the fact that the Coroner considers an inquest unnecessary," or form 99 "Coroner's certificate after inquest (deceased person)" would include those conditions not specified in the cause of death.

The present format for cause of death, 1a, lb, Ic and II would remain, as this is hallowed by tradition and accepted around the world, however subjective these entries might be. The ability to document other pathological processes would, in fact, make the task easier for all concerned, not least the Coroner's officer: "There's no more room Doc, the box is too small." The space provided for cause of death in fact measures $4 \frac{1}{2}$ " $\times 1 \frac{1}{2}$ " on Coroner's form 100 . The addendum proposed is denoted in the accompanying table and is suggested as a format for discussion. The information at present on the reverse of the non-Coroner's death certificate could well be included separately in the comprehensive introduction to each book of certificates as it only contains advice.

The certificate, completed on both sides, would be submitted either at the completion of the necropsy, or after any subsidiary tests become available - that is, histology, biochemistry, toxicology, etc. Many of the details proposed for completion could be filled in by an assistant, such as the Coroner's officer, during the necropsy.

\section{EXPLANATORY NOTES}

For simplicity and ease of documentation, what are considered common significant findings are itemised to be circled, the numbering being designed for computer retrieval. As most of the entries are positive findings there may be little to complete in an individual case. The usual sudden infant death - for example, might need only height and weight entered. It would be presumed atheroma was absent if the negative entries were not completed.

010-013 Height and weight of the body, in imperial or metric measurements, are obviously imperative, both for assessing cardiomegaly and for other reasons. Where scales or a linear measure are not available, the fact that either is estimated can also be recorded.

020-040 The "normal" size of the heart has intrigued many pathologists for years. Whether the tables appearing in textbooks of pathology are accurate or not would surely surface from these statistics. It would, moreover, appear rational to regard cardiomegaly, in the absence of any obvious clinically important disease process, as an important finding.

050-061 The distribution of atheroma and its association with sudden death is regarded as one of the most important features of this supplement to death certification. A clear and unambiguous definition of what should correspond to ++++++ needs to be formulated by a committee of experts.

12 "Significant other scarring" would include obvious old tuberculous lesions, pneumoconiosis, and pleural plaques, but it is not envisaged that pleural adhesions would be mentioned.

21 It is realised that oesophageal hernias or

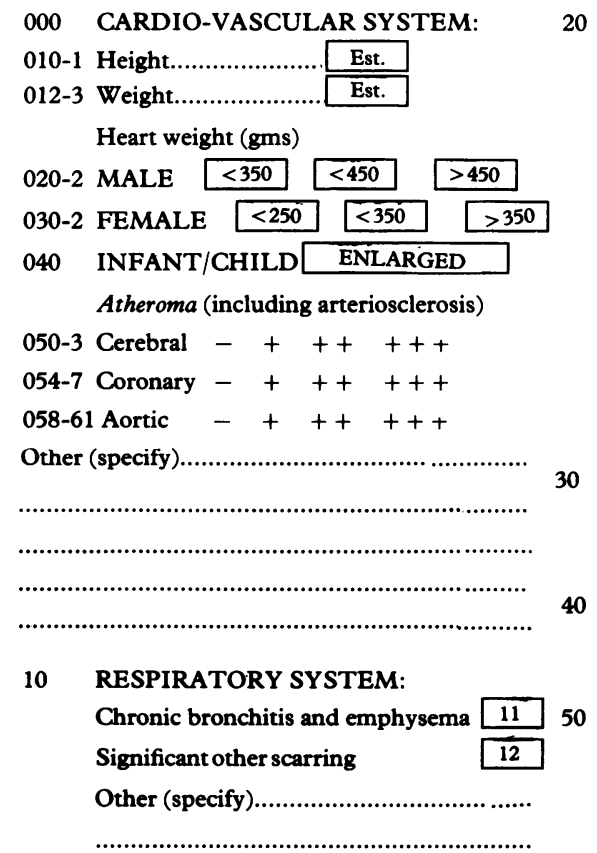

000 CARDIO-VASCULAR SYSTEM: Oesophageal hernia Ulcer gastric duodenal

Diverticulosis large bowel Cholelithiasis

Other (specify)

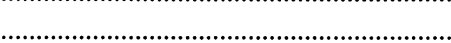

……………………

RETICULO-ENDOTHELIAL SYSTEM:

Specify...

ENDOCRINE SYSTEM:

Specify.

GENITO-URINARY SYSTEM:

Kidney scarring

Uterine fibroids

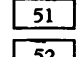

Other (specify)
60

CENTRAL NERVOUS SYSTEM:

Brain scarring

Other (specify)

70 DISEASES/CONDITIONS NOT

ITEMISED: (See explanatory notes)

Specify.

80

OPERATIONS PERFORMED:

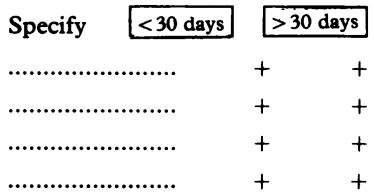

90-92 AUTOLYSIS PRESENT:

Mild Moderate Severe

93 (See explanatory notes) 
Barratt's oesophagus may be difficult to quantify in the presence of the usual post mortem changes, but it is felt worthwhile to evolve some definition for those lesions of the oesophagus above the diaphragm.

30 The absence of normal lymphadenopathy in infancy and childhood should be recorded, rather than its presence. Splenomegaly $(>250 \mathrm{~g}$ ) should perhaps be included?

40 It may be considered relevant to record adrenal hyperplasia, if only to show how common it is. Atrophy or enlargement of the thyroid might well also be considered to be itemised for circling.

50 It is not envisaged that simple ovarian cysts of less than $3 \mathrm{~cm}$ in diameter would be recorded, and it may be argued that the common fibroid also does not need to be itemised. Major kidney scarring may prove too difficult to document as the changes with age are so variable.

61 Brain scarring would include evidence of previous infarctions, haemorrhages, or brain operations.

70 In this space it is envisaged that congenital anomalies, inherited conditions, metabolic diseases, endocrine conditions, mental deficiency, ill defined conditions such as Parkinson's and dementia, should be included. The incongruity of not documenting a disease that may have no necropsy markers does not belie its importance-for example, hyperkalaemia, epilepsy. Cancers that have been treated and show no clinical evidence of their presence at necropsy are surely of the greatest importance. It would be expected that a death attributed to hyperglycaemic diabetic coma would have the supporting information in this section, such as blood sugar, urine results, either before or after death.

Unexpected histological findings, such as microscopically confirmed cancers, clinically important thyroiditides, manifestations in the brain or other organs, of chronic alcoholism, would be entered here. It is also suggested that space " 70 " might be referred to as the "honesty box" where a cause of death given as "bronchopneumonia" could be modified-for example, "histology revealed pneumonia not as widespread as grossly suggested," "a cardiac arrhythmia is the most likely mode of death," or "no adequate explanation for death." The medicolegal implications of such a statement would have to be addressed.

80 It is not proposed that "operations" would include those for minor lesions of skin or periphery, or those for trauma, unless considered pertinent. Itemisation would, incidentally, be invaluable to NCEPOD in checking the validity of their statistics.

90-92 Any deficiencies in recording details may be due to autolysis and need to be recorded.

93 This area is left vacant for any additional information that may be required from time to time. Any necropsy is a large potential source of research material. There are many substances that can be measured from blood or tissue samples. Routine blood alcohol estimations may show some surprising results.
Haemoglobin $A_{1} C$ as a monitor of diabetic control, vitreous urea and creatinine, serum cholesterol and many other substances, can all provide accurate information and useful epidemiological data in samples taken after death.

In this context the attitude of the public to the necropsy needs to be taken into account. There is always the danger of evoking irrational fears of "interfering with the body" or "assault of the dead" when he or she has "suffered enough." In a situation where medical and public opinion needs educating what could be more productive than mounting periodical investigations into the concentrations of toxic substances, such as carbon monoxide and lead in the body of a dead person? Documenting drugs that may be held to have an important impact on disease incidence is obviously an impracticable aim. It is not, however, difficult to envisage that a single drug (such as Aspirin) might be chosen for a year's survey to assess the prevalence of its use or influence on disease processes. Surgeons, with their NCEPOD survey, have shown a way forward in focusing on particular groups of deaths each year.

It can be observed from the above that many conditions could be itemised by medical staff signing death certificates without a necropsy having been done. Whether this task should be left to junior doctors is a subject that needs airing. General practitioners fill in certificates on their patients. When experienced pathologists find the task difficult after necropsy it is perhaps not unreasonable to suggest that our consultant hospital colleagues should be responsible for death certification where no necropsy has been performed. Consultants have complained to me that their junior staff do not request necropsies often enough; increased involvement by consultants would surely help. I know of one hospital where all death certification is overseen by a pathologist. Is this perhaps an appropriate lead to follow?

Agreement, in principle, for this proposal could open the door to the sensitive area of our approach to the Coroners and their area of jurisdiction. I cannot see that, with a little tact and diplomacy, any serious objection could arise as to what is, after all, an extension of the medical aspects of death certification.

\section{Conclusion}

Death certification is a vital source of demographic information. The necropsy and the information derived from it is as important today as it ever was. The great advances in the understanding of many diseases have not altered the fact that the human race still has a $100 \%$ mortality. The goalposts of disease incidence may well have moved but so have the errors. There can be no valid projections for the future if vital statistics are faulty. It is suggested that the additional information required would add veracity to death certification.

My thanks are due to our overworked secretarial staff, Mrs A Dunwood and Mrs R Vinicombe, for their help and assistance. 
1 Hill RB, Anderson RE. The Autopsy-medical practice and public policy. London: Butterworths, 1988.

2 Kircher T, Nelson J, Burdo $H$. The autopsy as a measure of accuracy of the death certificate. $N$ Engl J Med 1985 313:1263-9.

3 Goldman L, Sayson R, Robbins S, Cohn LH, Bettman M Weisberg $M$. The value of the autopsy in three medica eras. $N$ Engl J Med 1983;308:1000-5.

4 Editor's reply. Physician deaths under the age of 50 years. Information from obituaries. $J A M A$ 1988;260:3433.

5 Morley AR. Value of the autopsy. Association of Clinical Pathologists Newsletter 1988;9-13.

6 Underwood JCE, Cotton DWK, Stephenson TJ. Audit and necropsy. Lancet 1989;i:442.
7 Cameron HM, McGoogan E, Watson H. Necropsy as a yardstick for clinical diagnoses. Br Med J 1980;281:985-8 8 King DW. Potential of the autopsy. Arch Pathol Lab Med 1984;108:39-43.

9 Anderson RE, Fox R, Hill RB. Medical uncertainty and the autopsy: occult benefits for students. Hum Pathol 1990;21:128-35.

10 Anderson NH, Shanks JH, McCluggage GWG, Toner PG. Jecropsies in clinical audit. J Clin Pathol 1989;42: 897-901.

11 Campling EA, Devlin HB, Lunn JN. The report of National Confidential Enquiry into Perioperative Deaths 1989. London: Royal College of Surgeons, 1990.

\section{Eponyms in pathology...}

SALMON, Daniel Elmer (1850-1914) was an American veterinary pathologist. He was born in Mount Olive, New Jersey and studied at Cornell university, where he graduated in 1872. He was a practising vet in Newark, New Jersey until 1879 when he moved to the US Department of Agriculture in Washington DC, working first as an investigator and later (1884) as the first director of the newly formed Bureau of Animal Industry. In 1905 he became head of the national veterinary school in Montevideo where he remained for six years until 1912. In 1886 he discovered the non-lactose fermenting bacilli responsible for typhoid and paratyphoid fever (Salmonella sp), but among his other contributions was the discovery in 1886 (with Theodore Smith) that bacteria could produce protective immunity despite having been killed by heat. He died of pneumonia at Butte, Montana, aged 64. 